Running Head: SPATIOTEMPORAL ORGANIZATIONS IN WHITE MATTER

\title{
Latency structure of BOLD signals within white matter in resting-state fMRI
}

\author{
Bin Guo ${ }^{\text {a,b,* }}$, Fugen Zhou ${ }^{\mathrm{a}}$, Muwei Li ${ }^{\mathrm{b}}$, John C. Gore ${ }^{\mathrm{b}, \mathrm{c}, \mathrm{d}, \mathrm{e}, \mathrm{f}}$ \\ ${ }^{a}$ Image Processing Center, School of Astronautics, Beihang University, Beijing 100191, Beijing, \\ China \\ ${ }^{\mathrm{b}}$ Vanderbilt University Institute of Imaging Science, Vanderbilt University, Nashville, TN 37232, \\ USA \\ ${ }^{\mathrm{c}}$ Department of Radiology and Radiological Sciences, Vanderbilt University Medical Center, \\ Nashville, TN 37232, United States \\ ${ }^{\mathrm{d}}$ Department of Biomedical Engineering, Vanderbilt University, Nashville, TN 37232, United States \\ ${ }^{\mathrm{e}}$ Department of Molecular Physiology and Biophysics, Vanderbilt University, Nashville, TN 37232, \\ United States \\ ${ }^{\mathrm{f}}$ Department of Physics and Astronomy, Vanderbilt University, Nashville, TN 37232, United States
}

Key words: BOLD; fMRI; functional connectivity; resting state; white matter

Email addresses co-authors

Bin Guo: guobin420@gmail.com

Fugen Zhou: zhfugen@ buaa.edu.cn

Muwei Li:m.li@vumc.org

John C. Gore: john.gore@vumc.org

Corresponding author:

Bin Guo

Image Processing Center,

School of Astronautics,

Shahe Campus,

Beihang University,

No. 9, Nansan Road,

Shahe High Education Park,

Changping District,

Beijing, 102206

Phone:+86-18810601012

Email: guobin420@gmail.com 


\section{Dear Editor,}

Dynamics of the brain were studies from different imaging scales, including $\mathrm{Ca}^{2+}[1]$ cells [2], and brain regions [3]. In the research area of the dynamics between brain regions, functional magnetic resonance imaging (fMRI) studies based on the detection of changes in blood oxygenation-level dependent (BOLD) signals after a task or stimulus were the mostly used. The technique was later extended to include the detection and analysis of spontaneous signal fluctuations [4]. Though initially regarded as noise, these spontaneous fluctuations were later analyzed to reveal intrinsic synchronizations of regions within the somatomotor system of the brain [5], and these result in long range, inter-regional low frequency temporal correlations in measured BOLD signals. Conventional analyses of these temporal correlations yields a metric widely referred to as functional connectivity (FC) which is derived by assuming exact synchronization of activities and BOLD signals in intrinsic brain networks. However, previous studies in rat and humans found that BOLD fluctuations are spatio-temporally organized across different brain circuits [3,6]. To characterize the spatiotemporal patterns of brain activities, Mitra and colleagues recently analyzed inter-regional time latencies or lags in BOLD signals. They derived a time delay matrix from those lags corresponding to extrema in the cross-correlation functions of pairs of voxels. A highly reproducible, state-dependent latency projection (the average timing with respect to other voxels) spanning around 1 second, was found to correspond to spatially segregated functional networks $[7,8]$. Since then, much effort has been paid to comprehensively explore lead/lag relationships within the cerebral cortex [7], between the thalamus and the cerebral cortex [8], and the cerebellum [9]. Such relationships are important for appropriate data analysis and for the interpretation of functional interactions within and between networks.

Despite converging evidence for the functional role of BOLD signals in white matter [10-12], temporal properties of these signals have not been adequately investigated. White matter connects various gray matter areas of the brain to each other, and is responsible for transducing neural activity between regions. In light of recent advances in modelling of how networks communicate [13], examination of the timing relationships within white matter is particularly compelling.

Here we first report studies of the latency structure of BOLD signals within white matter. The results are derived from the Brain Genomics Superstruct Project (GSP), a publicly available dataset consisting of a large cohort of healthy young subjects. We found that a highly reproducible latency projection also exists in white matter, as shown in Figure 1-A. The latencies ranged from $-0.1 \mathrm{~s}$ to $0.1 \mathrm{~s}$, with positive values indicating lags and negative values leads in timings of signal propagations. It can be seen that the distribution of the latencies was highly bilaterally symmetric. The white matter regions with the smallest and greatest lag values were the internal capsule (IC) and the posterior corona radiata (PCR), respectively. A gradual increase in lag value from the posterior thalamic radiation (PTR) to the optic radiation (OR) was also observed. The correlogram in Figure 1-B shows the pair-wise correlation coefficient among the ten separate subgroups. The correlation coefficient value ranged from 0.859 to 0.908 , which demonstrates high spatial similarities in latency projections across subgroups.

Second, to investigate the integration of resting-state functional networks (RSNs), K-means $(\mathrm{K}=8)$ clustering was applied on dataset-level FC matrix (Clustering result was shown in Figure $\mathrm{S} 1$ in the supplementary 
material). To establish anatomical correspondences, networks were named according to the largest WM fiber bundle they contained for clusters (see Table S1 in the supplementary material for details), including middle cerebellar peduncle (MCP), posterior limb of inter capsule (PLIC), retrolenticular part of internal capsule (RPIC), corpus callosum (CC), anterior corona radiata (ACR), posterior thalamic radiation (PTR), superior longitudinal fasciculus (SLF), and superior corona radiata (SCR). Latency analyses among these RSNs are displayed in Figure 1-C, wherein diagonal and off-diagonal latency blocks indicate patterns of intra- and inter-network communications, respectively. Contrasting with the results from GM [11] wherein no significant lead/lag relationships at the network level were identified between RSNs, latency analysis in WM revealed the existence of inter-RSN lead/lag relationship, as indicated by asterisks in Figure 1-D (one sample t-test on the mean value of each off-diagonal block, $\mathrm{p}<0.05$ with Bonferroni correction, $\mathrm{N}=28$ ), which suggested that propagations of BOLD signals between paired RSNs, indicated by each asterisk, were dominated by one direction despite the actual existence of reciprocal communications. Thus, a dominant direction (indicated by black arrows while the other direction was indicated by blue arrows in Figure 1-E), from inferior WM (including PLIC) to middle WM (including RPIC) and further to superior WM (including PTR, SCR, SLF, and PTR) was identified. However, comparatively, signal propagations among the RSNs from superior WM, including CC, PTR, SCR, SLF, and PTR, showed weaker directional dominance, as shown in the right of Figure 1-E. Working mode of the brain is deemed to be not merely reflexive but rather mainly intrinsic in keeping interacting with the environment [14]. Functional organizations of the brain are sustained by its intrinsic activities, the spatial coherence of which has been found to transcend different levels of consciousness [15]. From this perspective, the operational model of the brain is to collect and interpret information from the environment, and respond to it whenever necessary. In this interacting progress, the WM is responsible for transferring neural information between the peripheral nervous system and central nervous system (CNS), and within the CNS. The signal propagation along the inferior-superior direction we observed may suggest that during a resting-state, the dominant mode of brain operations is primarily to collect and interpret information from the environment rather than respond to it.

Third, to explore the functional significance of the latency structure in WM, we also contrasted latency projections obtained during different sensory states (using Beijing EOEC dataset II) by applying a linear mixed-effects analysis to study the main effect of the sensory states, along with the in-between paired effects. As shown in of Figure 2-A (only regions of statistical significance, defined by FWE-corrected $\mathrm{p}<0.05$ and uncorrected $\mathrm{p}<0.001$, were included), one prominent cluster was observed in the IC and another in the OR. Post hoc t-tests suggested significant altered latencies within these two regions between different sensory states, eyes closed (EC) vs. eyes open (EO) (middle panel in Figure 2-A), and $\mathrm{EC}$ vs. eyes open with fixation (EOF) (bottom panel in Figure 2-A). However, no significant regions were observed when contrasting EO and EOF. Notable differences in latency values among the three sensory states were also clearly visible. Larger lag values tended to be concentrated around the OR for EC as compared with EO (Figure 2-B), while small latency values tended to be around the IC for EO and EOF. Specifically, lag values within regions showing significant paired effect changed from $-0.46 \mathrm{~s} \sim 0.44 \mathrm{~s}$ in EC to $-0.16 \mathrm{~s} \sim$ $0.17 \mathrm{~s}$ in $\mathrm{EO}$, and lag values within regions of 
significant effect changed from $-0.46 \mathrm{~s} \sim 0 \mathrm{~s}$ in EC to $-0.14 \mathrm{~s} \sim 0.07 \mathrm{~s}$ in EOF, suggesting potentially altered levels of WM engagement in different sensory states.

These three findings combined provide additional insights into the characters and functional roles of white matter BOLD signals in brain functions (further discussions were included in the supplementary material).

\section{Author Conflicts}

The authors declare no competing interests.

\section{Acknowledgements}

This work was supported by the National Institu tes of Health(NIH) grant R01 NS093669 (J.C.G ) and R01 NS113832 (J.C.G). Also, we sincerely thank Dr. Zhaohua Ding for his insights in our discussion.

\section{References}

[1] Tang Y, Li L, Sun L, et al. In vivo two-photon calcium imaging in dendrites of rabies virus-labeled v1 corticothalamic neurons. Neuroscience bulletin, 2020, 36(5): 545-553.

[2] $\mathrm{Hu}$ Y, Shi P, Gao Z. Norepinephrine from the locus coeruleus regulates microglia dynamics during wakefulness. Neuroscience bulletin, 2020, 36(5): 554-556.

[3] Majeed W., Magnuson M., Keilholz S. D. (2009). Spatiotemporal dynamics of low frequency fluctuations in BOLD fMRI of the rat. Journal of Magnetic Resonance Imaging: An Official Journal of the International Society for Magnetic Resonance in Medicine, 30(2), 384-393.

[4] Fox M. D., Raichle M. E. (2007). Spontaneous fluctuations in brain activity observed with functional magnetic resonance imaging. Nature reviews neuroscience, 8(9), 700-711.

[5] Biswal, B., Zerrin Yetkin, F., Haughton, V. M., \& Hyde, J. S. (1995). Functional connectivity in the motor cortex of resting human brain using echo-planar MRI. Magnetic resonance in medicine, 34(4), 537-541.

[6] Majeed W., Magnuson M., Hasenkamp W., Schwarb H., Schumacher E. H., Barsalou L., Keilholz S. D. (2011). Spatiotemporal dynamics of low frequency BOLD fluctuations in rats and humans. Neuroimage, 54(2), 1140-1150.

[7] Mitra A, Snyder AZ, Hacker CD, Raichle ME (2014) Lag structure in resting-state fMRI. Journal of Neurophysiology. 111(11): 2374-2391.

[8] Mitra A, Snyder A Z, Blazey T, et al. (2015) Lag threads organize the brain's intrinsic activity. Proceedings of the National Academy of Sciences, 112(17): E2235-E2244.

[9] Marek S., Siegel J. S., Gordon E. M., Raut R. V., Gratton C., Newbold D. J., et al, A. (2018). Spatial and temporal organization of the individual human cerebellum. Neuron, 100(4), 977-993.

[10]Gawryluk J. R., Mazerolle E. L., D'Arcy R. C. (2014). Does functional MRI detect activation in white matter? A review of emerging evidence, issues, and future directions. Frontiers in neuroscience, 8, 239.

[11]Gore J. C., Li M., Gao Y., Wu T. L., Schilling K. G., Huang Y., Anderson A. W. (2019). Functional MRI and resting state connectivity in white matter-a mini-review. Magnetic resonance imaging, 63, $1-11$.

[12]Grajauskas L. A., Frizzell T., Song X., D'Arcy R. C. (2019). White matter fMRI activation cannot be treated as a nuisance regressor: Overcoming a historical blind spot. Frontiers in neuroscience, 13, 1024.

[13]Mitra A, Raichle M E. (2016) How networks communicate: propagation patterns in spontaneous brain activity. Philosophical Transactions of the Royal Society B: Biological Sciences, 371(1705): 20150546. 

available under aCC-BY-NC-ND 4.0 International license.

[14]Raichle M. E., (2010) Two views of brain function. Trends in cognitive sciences, 14(4): 180-190.

[15]Raichle M. E., (2011) The restless brain. Brain connectivity, 1(1): 3-12. 
